
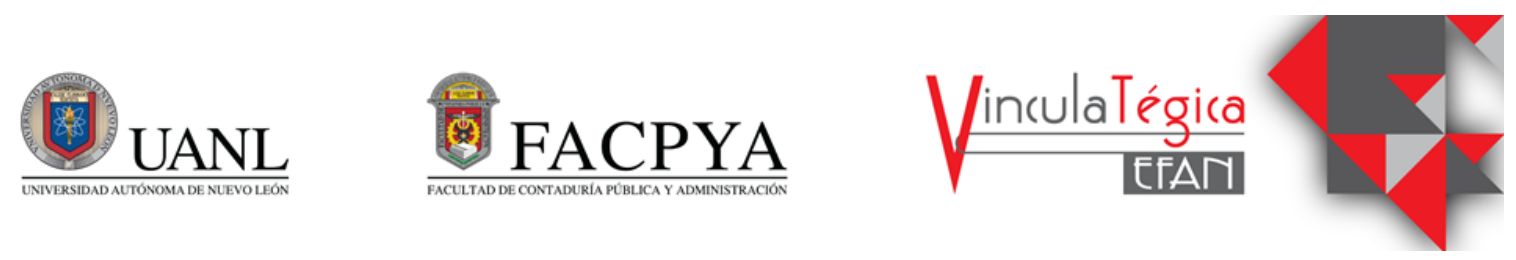

\title{
Percepción del impacto de las materias económico-administrativas en el emprendimiento, caso Facultad de Contaduría Pública BUAP
}

\author{
Blanca Hortencia Morales Vázquez ${ }^{1}$, Rebeca Muñoz Velázquez² y María de Jesús Ramírez \\ Domínguez $^{3}$
}

IBenemérita Universidad Autónoma de Puebla, blanca.morales.buap@gmail.com, Boulevard Capitán Carlos Camacho Espíritu No. 70. Col. Universidades C. P. 72590 Puebla, Pue. Tel: 2295500 Ext: 7670

${ }^{2}$ Benemérita Universidad Autónoma de Puebla, rebeca_mv@hotmail.com, Boulevard Capitán Carlos

Camacho Espíritu No. 70. Col. Universidades C. P. 72590 Puebla, Pue. Tel: 2295500 Ext: 7670

${ }^{3}$ Benemérita Universidad Autónoma de Puebla, mjrd_2001@yahoo.com, Boulevard Capitán Carlos Camacho Espíritu No. 70. Col. Universidades C. P. 72590 Puebla, Pue. Tel: 2295500 Ext: 7670

\author{
Información del artículo revisado por pares \\ Fecha de aceptación: junio-2021 \\ Fecha de publicación en línea: diciembre-2021 \\ DOI: https://doi.org/10.29105/vtga7.1-132
}

\section{Resumen}

El presente trabajo de investigación tiene por objetivo explorar el grado de conocimientos que tiene el estudiante de la Facultad de Contaduría Pública (FCP) de la Benemérita Universidad Autónoma de Puebla (BUAP), sobre el emprendimiento actual en México, América Latina y el mundo. La caracterización del fenómeno emprendedor es una descripción a través de variables observables tanto del emprendedor como del emprendimiento potencial y más aún ante el nuevo escenario que nos presenta la actual pandemia resultado del Covid-19. Por lo tanto, la universidad y los docentes juegan un papel de suma importancia en la formación de emprendedores. Por lo tanto, es posible identificar elementos con influencia significativa que impactan en el emprendedor de la Facultad de Contaduría Pública de la BUAP, lo anterior como consecuencia natural del diseño de la currícula de la Facultad. Los hallazgos encontrados en esta primera fase de investigación y la representación cualitativa y cuantitativa de los mismos, se encuentran plasmados en este trabajo de investigación como los resultados arrojados sobre la percepción que acerca del emprendimiento existe en la muestra elegida de los estudiantes de la Facultad de Contaduría Pública.

Palabras clave: L26 Emprendimiento, M13 Creación de empresas, O31 Innovación

\begin{abstract}
This research aims to explore the degree of knowledge that the student of the School of Public Accounting of the Benemerita University has about the actual entrepreneurship in Mexico, Latin America and the world. The characterization of the entrepreneurial phenomenon is a description through observable variables of both the entrepreneur and the potential entrepreneurship and even more so in the face of the new scenario presented by the current pandemic as a result of Covid-19. Therefore, the university and teachers play a very important role in the training of entrepreneurs. Therefore, it is possible to identify elements with significant influence that impact the entrepreneur of the School of Public Accounting of the BUAP, the above as a natural consequence of the design of the curriculum of the School.The findings found in this first phase of research and the qualitative and quantitative representation of them are reflected in this research as the results of the perception of entrepreneurship in the selected sample of students from the School of Public Accounting.
\end{abstract}

Keywords: L26 Entrepreneurship, M13 Entrepreneurship, O31 Innovation 


\section{INTRODUCCIÓN}

El emprendimiento constituye, hoy por hoy, una estrategia para el desarrollo económico y social de los países y de las regiones en la medida en que aprovecha y promueve la iniciativa de los individuos o de pequeños grupos con un alto deseo de independencia económica y sentido de logro, para generar un círculo virtuoso de prosperidad, innovación, crecimiento y desarrollo.

El mayor problema es que, aunque los emprendedores son el motor de la economía mexicana, por su mala gestión financiera el $80 \%$ fracasan en el país antes de los cinco años, y $90 \%$ antes de cumplir una década.

El profesionalizar al emprendedor desde su formación debe ser un compromiso que se asuma desde la promesa misma que se le hace al estudiante en el momento en que se le está ofreciendo un programa educativo con cierto perfil de egreso, por lo cual afinar, fortalecer o reorientar el Programa Educativo sería lo mínimo que garantice su inserción laboral.

Este trabajo de investigación tiene por objetivo el explorar el grado de conocimientos que tiene el estudiante de la Facultad de Contaduría Pública (FCP) de la Benemérita Universidad Autónoma de Puebla (BUAP), sobre el emprendimiento actual en México, América Latina y el mundo.

La principal conjetura que se tiene al respecto de este planteamiento del problema es, sin duda, que si logramos detectar los nuevos criterios del emprendimiento en el escenario que actualmente se está viviendo, entonces se podrán detectar a tiempo los cambios necesarios de las materias económico administrativas en el emprendimiento de la Facultad de Contaduría Pública de la BUAP para impactar la percepción del estudiante referente a este tema e inclusive mejorar la inserción laboral de nuestros egresados.
Comprender el concepto de emprendimiento es muy amplio, ya que existen muchas definiciones dependiendo de la connotación que se le adjudique:

existen diversas formas de definir y entender el término emprendedor; sin embargo, los más variados autores coinciden en que tal vocablo se deriva de la palabra entrepreneur, que a su vez se origina del verbo francés entreprendre, que significa encargarse de (Alcaraz, 2011 p. 21)

Más recientemente, el emprendimiento no solo se relaciona con la creación de una empresa producto de una persona visionaria que pone en marcha todos sus recursos para autoemplearse y convertirse, en algunos casos en fuente de trabajo para otros, sino que va más allá y es ofrecer nuevos productos o servicios con valor agregado, es fortalecer la competencia del liderazgo para dirigir a su nuevo equipo de trabajo, es tener claridad en el rol de desempeño de cada uno de los que conforman a la nueva empresa, es exponerse a los grandes retos que conlleve la misma, es hacer frente a grandes transnacionales que cuentan con demasiados recursos, asimismo, cuidando la eficiencia, la efectividad, el comportamiento en el desarrollo de nuevos proyectos, conllevando el emprendimiento a una planeación sin descuidar la estrategia, la creatividad, etc.

Por lo anterior, conocer las características de un emprendedor nos podrán permitir comprender mejor el concepto, por lo que en la Figura 1, se señalan las que con mayor recurrencia destacan:

\section{MARCO TEÓRICO}


Figura 1. Características del emprendedor Características del emprendedor

\begin{tabular}{l}
\hline Compromiso total, determinación y perseverancia \\
\hline Capacidad para alcanzar metas \\
\hline Orientación a las metas y oportunidades \\
\hline Iniciativa y responsabilidad \\
\hline Persistencia en la solución de problemas \\
\hline Realismo \\
\hline Autoconfianza \\
\hline Altos niveles de energía \\
\hline Busca de realimentación \\
\hline Control interno alto \\
\hline Toma de riesgos calculados \\
\hline Baja necesidad de estatus y poder \\
\hline Integridad y confiabilidad \\
\hline Tolerancia al cambio
\end{tabular}

Fuente: Elaboración propia con información de Kao, J., (1989).

Herrera y Montoya (2013), señalan que el aumento en las cifras reportadas en la base de datos de Scopus respecto al interés por parte de los investigadores en el tema de emprendimiento, se ha ido incrementando, ya que de 2008 a 2009 el número de publicaciones sobre dicho tema ha pasado de 862 a 1024 y en 2011 a 1497. (p.14)

El término emprendedor en un sentido económico fue usado, por primera vez, en el siglo XVIII por Richard Cantillon para describir al agente que asume riesgos en el mercado para combinar factores productivos y ofrecer un nuevo producto.

Inclusive, cabe señalar que ha habido una concepción incorrecta entre lo que es el emprendedor, un inversionista o un empresario, sin embargo, hay características muy particulares de cada uno de ellos que manejar el término como sinónimo es una equivocación.

Según Schollhammer (1980) divide a los emprendedores en cinco tipos de personalidades.

El emprendedor administrativo. Hace uso de la investigación y del desarrollo para generar nuevas y mejores formas de hacer las cosas.

El emprendedor oportunista. Busca constantemente las oportunidades y se mantiene alerta ante las posibilidades que le rodean.

El emprendedor adquisitivo. Se mantiene en continua innovación, la cual le permite crecer y mejorar lo que hace.

El emprendedor incubador. En su afán por crecer y buscar oportunidades y por preferir la autonomía, crea unidades indispensables que al final se convierten en negocios nuevos, incluso a partir de alguno ya existente.

El emprendedor imitador. Genera sus procesos de innovación a partir de elementos básicos ya existentes, mediante la mejora de ellos. (p. 24)

Sin embargo, al comparar la literatura acerca del tema la mayoría de los autores coincide en que para que el emprendimiento sea verdaderamente un impulsor del desarrollo económico de los países debe ser un emprendimiento con innovación que genere y agregue valor. Por lo tanto, hoy el termino emprendedor tiene una profunda relación con la innovación.

Banco Mundial realizó un estudio que con el puro título nos lleva a la reflexión: "El emprendimiento en América Latina. Muchas empresas y poca innovación", es decir estamos estancados en las formas económicas del siglo pasado (o antes de eso) y lo que las "potencias latinoamericanas" (Argentina, Brasil, Chile, Colombia y México) destinan para investigación y desarrollo en conjunto, es apenas una tercera parte de lo que China dedica al mismo rubro. Tal parece que se nos hubiera olvidado la capacidad de innovar, o peor aún, que la hubiéramos perdido. 
La anterior cita invita a la reflexión reafirmando que América Latina requiere emprendimiento con innovación, para lograr trascender y tener un impacto económico. Para América Latina el momento de ser exportadores de materias primas, ser poco productivos y generar poco valor ha pasado, Omar Zarur, director general Amway de México y Centroamérica destaca que el emprendimiento en México se ve favorable, pese a los cambios políticos y económicos pues "La propuesta de emprendimiento no depende del ambiente político ni económico, sino del chip del individuo por querer convertirse en emprendedor." Esta idea deja en claro que cualquier persona que genuinamente posea el talento de emprendedor continuará en ese camino, aunque tenga que sortear obstáculos de todo tipo.

Por otra parte la Comisión Económica para América Latina y el Caribe (CEPAL) en el documento Perspectivas económicas para América Latina 2017, afirma que sin duda el emprendimiento es un vehículo para promover la empleabilidad y la movilidad social de los individuos sin embargo reconoce que en los países de América Latina los jóvenes emprendedores tienen condiciones más adversas respecto de los de los países pertenecientes a la Organización para la Cooperación y el Desarrollo Económico (OCDE) tienen menos nivel educativo, han desarrollado menos competencia y experiencia, tienen menos recursos y es más difícil acceder al financiamiento. Existen obstáculos más marcados como el superar las barreras regulatorias y entender las complicadas legislaciones fiscales, así como adquirir las competencias empresariales. Las políticas Públicas en materia de emprendimiento han tenido una ligera mejoría, sin embargo, no han sido suficientes y en el caso de México con la desaparición del Instituto Nacional del Emprendedor el 10 de abril de 2019 hay un retroceso, porque dicho Instituto propiciaba un ecosistema emprendedor.

Las instituciones de educación superior deberán ser capaces de responder a los cambios que se están presentando en el mercado laboral: mayor competencia por empleos dignos y bien remunerados; flexibilización, precarización e informalidad laboral; pérdida de estabilidad y seguridad en el empleo; creciente rotación de puestos de trabajo; mayores exigencias de calificación y capacidad para crear organizaciones productivas para auto emplearse y a la vez generar empleo (Asociación Nacional de Universidades e Instituciones de Educación Superior [ANUIES], 2016, p. 24).

Continuando con las aseveraciones de investigadores de América Latina que han medido variable de la formación de emprendedores han mostrado en su resultados que:

La formación de los recursos humanos es deficiente $\mathrm{y}$, además, el sistema educativo no prepara los talentos necesarios. Significa que no existe una masa crítica de investigadores e innovadores, capaces de crear empresas eficientes y sostenibles, incorporar las nuevas tecnologías y propulsar el cambio productivo. El resultado es un rezago educativo que lastra el capital humano y reduce las posibilidades de progresar en innovación y emprendimiento (Arancibia, 2021, p. 23). 
Finalmente, tal como lo señala MababuMukiur (2017), en su artículo "La transformación digital y el emprendimiento de los jóvenes en Iberoamérica" para enfrentar los actuales desafíos del mercado, las universidades deben desarrollar habilidades en el emprendimiento de los jóvenes en el contexto actual de la transformación digital que implica no solo la incorporación de las tecnologías emergentes, sino también nuevos modelos de negocio más ágiles y eficaces, ya que se pone de manifiesto que la transformación digital abre nuevo espacio de negocios en los países iberoamericanos y promueve el emprendimiento de los jóvenes para la transformación social y económica (p.1)

\subsection{Lo inesperado: La pandemia sufrida por el Covid-19}

El 31 de diciembre de 2019 la Oficina de la Organización Mundial de la Salud, OMS, (2021) en la República Popular China detecta una declaración de la Comisión Municipal de Salud de Wuhan para los medios de comunicación publicada en su sitio web en la que se mencionan casos de una «neumonía vírica» en Wuhan (República Popular China). La OMS solicita a las autoridades chinas información sobre el conglomerado de casos de neumonía atípica en Wuhan del que ha tenido noticia. En una corta línea de tiempo se descubre que el virus atípico es COVID-19. El 16 de enero de 2020 se publica la primera alerta epidemiológica y se empieza a propagar la infección por todo el mundo llegando a la inminente situación de declarar una pandemia. Con todo esto, comisiones a nivel mundial en coordinación con los gobiernos comienzan a trabajar en directrices para la planificación operacional en apoyo de la preparación y la respuesta de los países. Entre las muchas directrices, la fuerte campaña de "quédate en casa" obligó a contener a las más personas posibles en una cuarentena casi obligada para evitar contagios masivos además de que la mayoría de los gobiernos de diferentes países tomaron la decisión de decretar el cierre de comercios no esenciales para evitar conglomeraciones innecesarias que fueran focos de infección que detonara, de igual forma, más contagios, sin embargo, el resultado de todo esto conllevo a que las economías de los países se ralentizaron (por no decir se pararon), esto es, las medidas de bloqueo como respuesta a la crisis han frenado aún más las actividades económicas a nivel mundial.

En este contexto destaca la importancia del papel de los emprendedores para acelerar la recuperación económica, entonces se vuelve de vital importancia analizar cómo los emprendedores enfrentan la situación causada por el COVID-19, ya que estas nuevas circunstancias los han enfrentado a desafíos como la necesidad de digitalización, la falta de financiamiento, la disminución del capital humano o la percepción de nuevas oportunidades que se conviertan en negocios viables.

Es un hecho que la crisis originada por el COVID-19, la cuarentena y el temor a salir de casa, así como la revolución de la tecnología; se han convertido en generadores de cambios en la forma de hacer negocios, en concreto se ha vuelto todo un reto las ventas físicas, situación que ha llevado a los emprendedores tradicionales a incursionar en el E-Commerce o el comercio electrónico o el comercio digital; siendo esta una herramienta imprescindible para realizar transacciones de compra y venta de productos en forma virtual.

Entonces queda claro que, con esta nueva realidad se acorta la brecha hacia lo digital, de un golpe estamos inmersos en clases virtuales, comercio digital, teletrabajo, todo en línea, por lo que se vuelve vital y prioritario analizar, para fines de este trabajo, cómo impactaría dicha situación a los emprendedores 
tradicionales y cómo impactar la percepción del estudiante de las materias económico administrativas en el emprendimiento de la Facultad de Contaduría Pública de la BUAP para que su visión sea acorde a este nuevo contexto.

\section{MÉTODO}

El método empleado fue documental, descriptivo y de campo, en las primeras dos se revisó material de fuentes primarias y secundarias (journals especializada e instituciones como la OCDE, etc.), para la investigación de campo se estudió a una población de 3000 alumnos con una muestra de 700, información que permitió analizar y describir cualitativa y cuantitativamente el grado de conocimientos que tiene el estudiante de la FCP de la BUAP, sobre el emprendimiento actual en México, América Latina y el mundo, esto es, con la aplicación de un instrumento se pudo explorar y conocer la percepción del impacto que tienen las materias económico-administrativas en la formación del emprendedor y al conocer los resultados poder estar en la posibilidad de aportar mejoras a la currícula y estrategias de mejora para fortalecer el talento emprendedor de los estudiantes de la Facultad de Contaduría Pública.

Por lo anteriormente expuesto, poco a poco se están dando los andamiajes necesarios para transitar a una sociedad con emprendedores innovadores por esa razón en la Facultad de Contaduría Pública de la Benemérita Universidad Autónoma de Puebla, para abonar a fomentar el emprendimiento innovador, como primera fase se está elaborando esta investigación que plantea indagar qué conocimientos tienen los estudiantes acerca del entorno emprendedor, la forma en la que perciben el emprendimiento en América Latina y el mundo así como el impacto de las materias económico administrativas en el perfil emprendedor. Para lo cual se aplicó un instrumento a una muestra de 700 estudiantes de una población de 3000 , dicho instrumento contiene 20 ítems de los cuales se presentan los más relevantes.

Figura 2. Significado de las siglas IEG 1.- A continuación, se menciona el significado de las siglas IEG, selecciona la respuesta correcta.

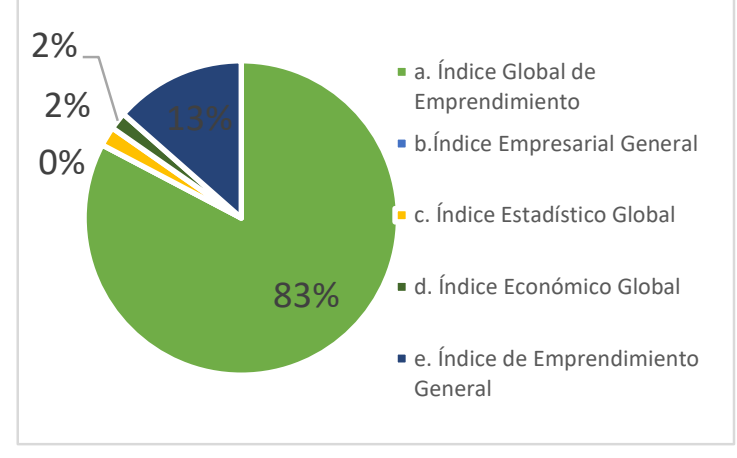

Fuente: Elaboración propia

De acuerdo con Global Entrepreneurship Index 2018, en español "Índice Global de Emprendimiento" (IEG) es un indicador que se compone de la salud del ecosistema de emprendimiento en un país determinado. Los resultados de la aplicación de la encuesta muestran que $82.7 \%$ de los encuestados saben el significado correcto, el $13.5 \%$ indico que Índice de Emprendimiento General era el correcto y el $3.8 \%$ respondieron de manera errónea. En general podemos decir que el $17.3 \%$ de los alumnos encuestados desconoce el significado de las siglas mencionadas.

\section{Figura 3. Qué mide el índice global de} emprendimiento

2.-De los siguientes indicadores, ¿qué es lo que mide el índice global de emprendimiento? 


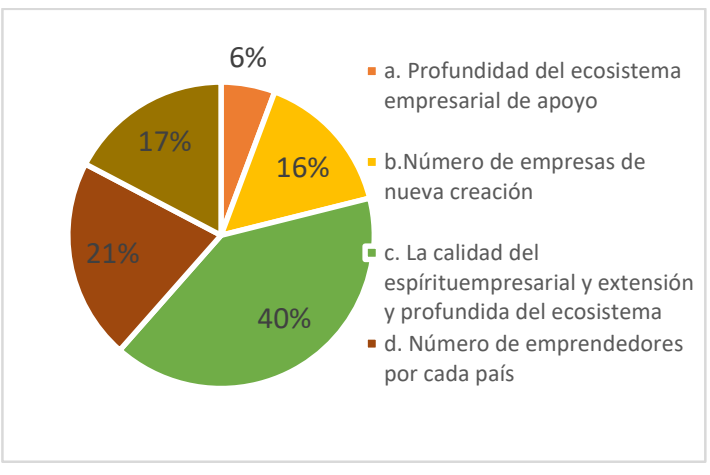

Fuente: Elaboración propia

El IEG es el encargado de medir la calidad del espíritu empresarial, la extensión y profundidad del ecosistema empresarial de apoyo y está conformado por 14 componentes denominados GEI. Los datos que arrojan la encuesta muestran que el $5.7 \%$ puso como respuesta uno de los indicadores, 53.9\% mencionó otros indicadores incorrectos y solo el $40.4 \%$ conoce el objetivo principal de este índice.

Figura 4. País número 1 en emprendimiento 3.-De acuerdo con el ranking del Îndice Global de Emprendimiento ¿Sabes cuál es el país número 1 en emprendimiento?

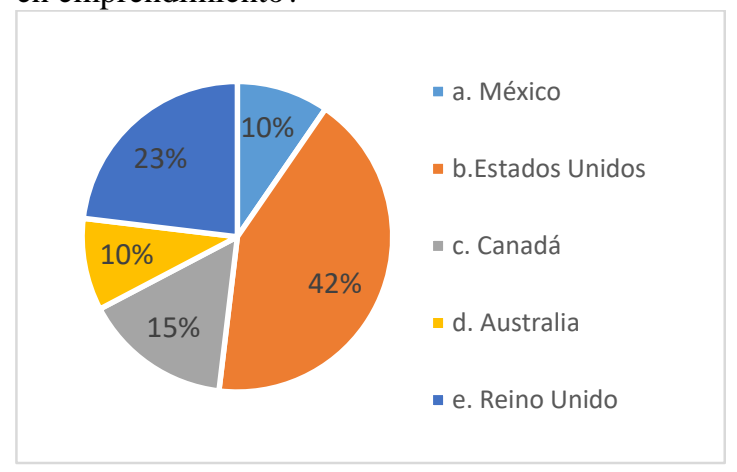

Fuente: Elaboración propia

El ranking en el IEG se mide a través del GEI conformado por 14 componentes. El 9.6\% menciona que México, pero nuestro país ocupo la posición 75, continuando con la estadística 9.6\% respondió que era Australia, sin embargo, se encuentra en la quinta posición a quien le antecede Reino Unido con la cuarta y Canadá con la tercera, finalmente el
42.3\% sabe que Estados unidos se encuentra en lugar número 1 del Global Entrepreneurship Index 2018.

Figura 5. Mejores países para emprender 4.-De los Países que se muestran ¿Sabes cuáles son los mejores para emprender?

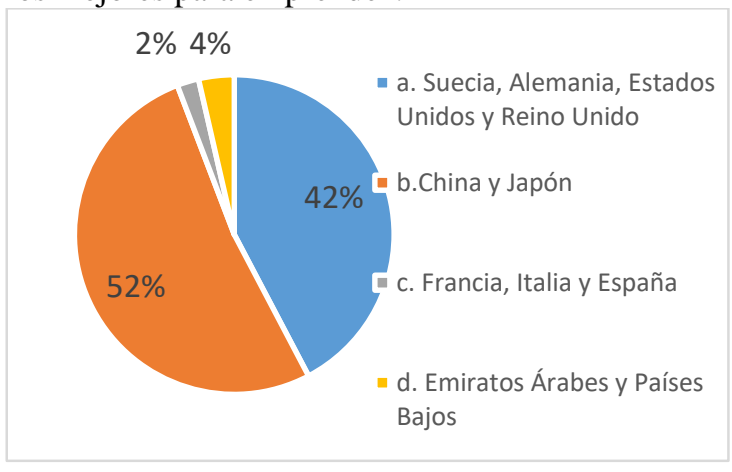

Fuente: Elaboración propia

Más del 50\% de los encuestados asegura que China y Japón son los mejores países para emprender, pero Lindsey Galloway en su artículo "Cinco Países Preparados para el Éxito de una Start-up" publicado el 9 de abril 2018 en la revista BBC Worldwide menciona que los cinco países son Suecia, Alemania, Estados Unidos y Reino Unido, el $42.3 \%$ de los participantes de la encuesta coinciden con ella.

Figura 6. Áreas de emprendimiento más fuerte de América del norte, IEG

5.- De las áreas de emprendimiento enlistadas ¿Cuál crees que sea la más fuerte de América del norte el Î́ndice Global de Emprendimiento? 


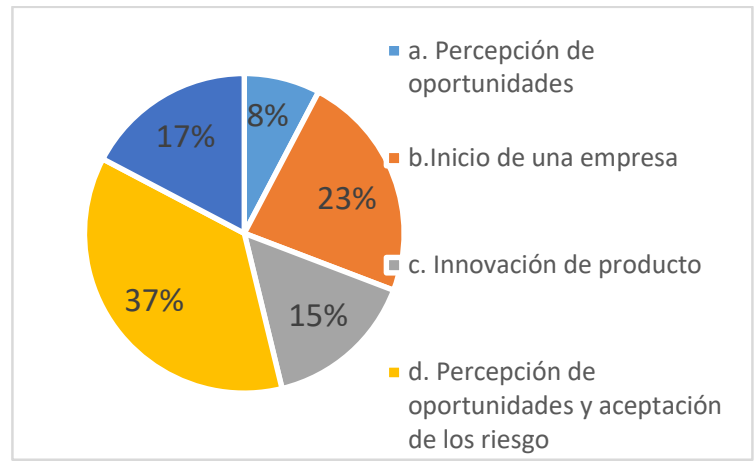

Fuente: Elaboración propia

El Índice Global de Emprendimiento 2018 señala que la región de América del Norte presenta una mayor fuerza en las áreas de Percepción de Oportunidades y Aceptación del Riesgo, esto quiere decir que los emprendedores son capaces de detectar y actuar sobre las mejores oportunidades para iniciar un negocio. Solo el $36.5 \%$ señaló de manera correcta, el $63.5 \%$ tiene ideas diversas a la realidad.

Figura 7. Principal factor del lento crecimiento de las empresas de América Latina

6.-De acuerdo con la percepción que tienes sobre la economía regional, ¿Cuál crees que sea el factor principal del lento crecimiento de las empresas de América Latina?

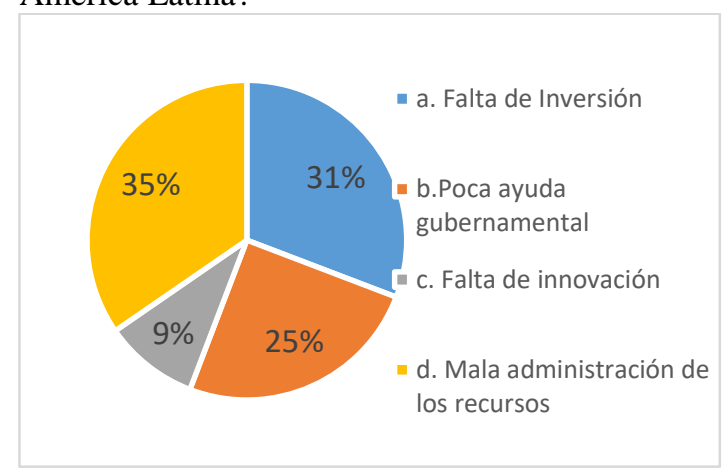

Fuente: Elaboración propia

En la obra "El Emprendimiento en América Latina, Muchas Empresas Poca Innovación" realizada por Daniel Lederman, Julián Messina, Samuel Pienknagura y Jamele Rigolini. Afirman que hay una serie de factores que impiden el crecimiento de las empresas Latino Americanas, pero principalmente se debe a la falta de innovación al igual que invierten muy poco en Investigación y desarrollo. Del total de las respuestas de los encuestados solo el $9.6 \%$ señaló como respuesta la falta de innovación mientras que el $34.6 \%$ piensa que es la mala administración de los recursos, el 30.8\% la falta de inversión y el $25 \%$ asegura que se debe al poco apoyo gubernamental.

Figura 8. Sobrevivencia de empresas de América Latina en mercados de exportación

7-¿Sabes qué tiempo sobreviven las empresas de América Latina que entran a los mercados de exportación?

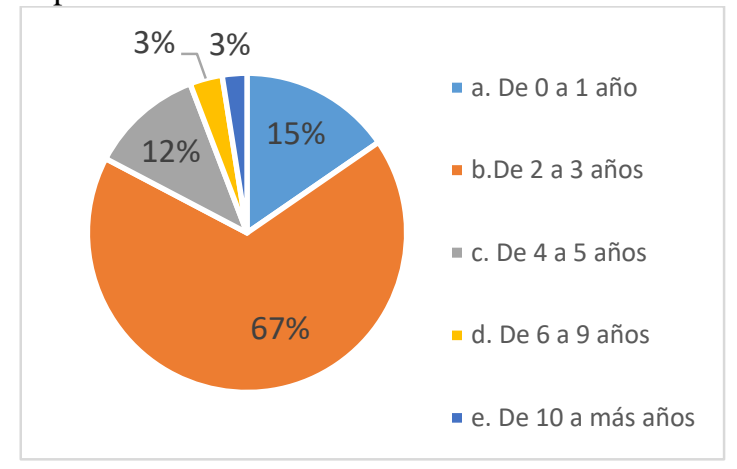

Fuente: Elaboración propia

Las empresas de América Latina que entran a los mercados de exportación no sobreviven más de un año, si bien las empresas que deciden entrar al mercado de exportación presentan una gran capacidad para adaptarse y lidiar con la adversidad, no es suficiente para trascender en los mercados extranjeros. Más del $50 \%$ de los encuestados cree que las empresas tienen una vida en los mercados de 2 a 3 años y solo el $15.4 \%$ conoce las estadísticas.

Figura 9. México en el ranking del Índice Global de Emprendimiento 
8.-De las siguientes posiciones, ¿Cuál ocupa México en el ranking del IEG?

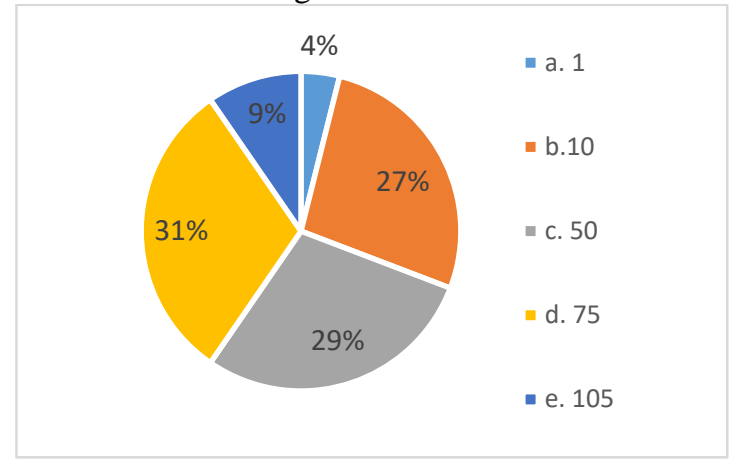

Fuente: Elaboración propia

México en el Índice Global de Emprendimiento ocupa la posición número 75 destacando en las áreas de Networking e Innovación de Producto y en el ranking lo supera Chile, Puerto Rico, Colombia, Uruguay, Barbados, Costa Rica, Belice, Perú, Panamá y Trinidad y Tobago respectivamente. Solo $30.8 \%$ conoce la posición de su país, el resto tiene ideas diferentes.

Figura 10. Políticas públicas para el emprendimiento

9.- A continuación, se menciona una serie de recursos necesarios para el emprendimiento, ¿Cuál de estos crees que les hace falta a las políticas públicas del actual gobierno, en esta materia?

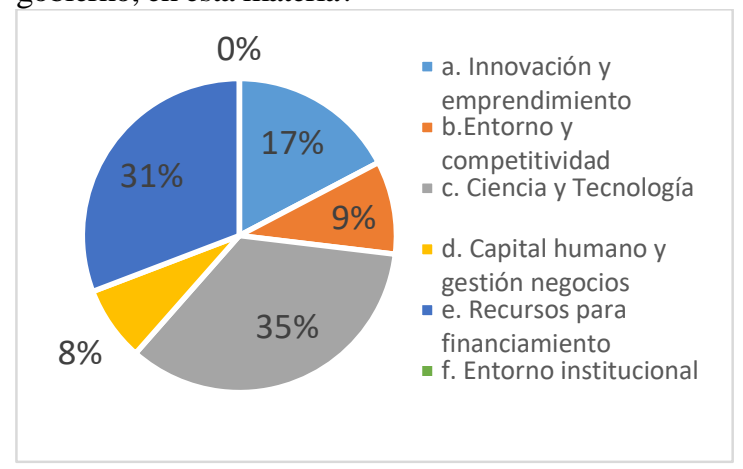

Fuente: Elaboración propia

De acuerdo con Gómez y Mitchell (2014) el pilar básico del emprendimiento es el entorno institucional y a ello se le suman los demás recursos mencionados en esta pregunta, sin embargo, otros autores difieren en que éste sea la base. Las respuestas más seleccionadas fueron Ciencia y Tecnología con un $34.6 \%$ y Recursos para Financiamiento un $30.8 \%$ con esto podemos decir que las políticas públicas que debe implementar el gobierno actual de nuestro país deben ir enfocadas a la generación de incentivos para que las empresas destinen mayores recursos a la investigación, ciencia y tecnología seguidos de la innovación.

Figura 11. Modalidades de apoyo del gobierno federal al emprendimiento

10.-¿Sabes cuáles son las modalidades de apoyo del gobierno federal al emprendimiento?

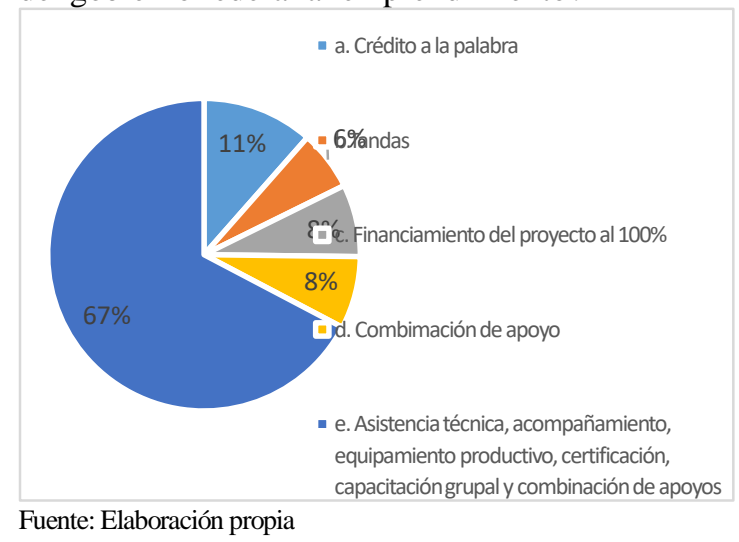

El Gobierno Federal de México ofrece diferentes modalidades de apoyo a los emprendedores, sin embargo, sólo el $67.3 \%$ de los encuestados contestó correctamente y el resto de los encuestados desconoce de dichas modalidades.

Figura 12. Solicitudes de apoyo del gobierno en materia de emprendimiento 11. ¿Alguna vez has solicitado apoyo del gobierno en materia de emprendimiento? 


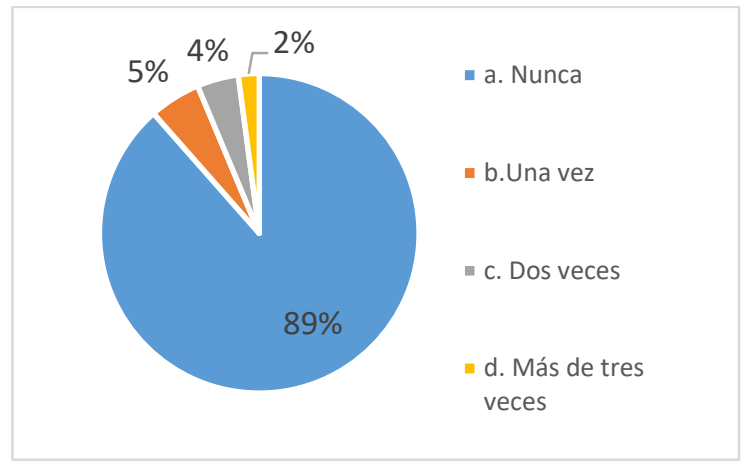

Fuente: Elaboración propia

El Gobierno Federal de México siempre ha ofrecido apoyos a los emprendedores y de los encuestados el $88.5 \%$ no ha solicitado ningún tipo de apoyo gubernamental en materia de emprendimiento, solo el $11.5 \%$ ha solicitado una o más veces y solo el $3.8 \%$ fue beneficiado.

\section{RESULTADOS.}

Los hallazgos encontrados en esta primera fase de investigación y de la representación cualitativa y cuantitativa de los mismos se encuentran plasmados en este trabajo, tal como se muestra en la recolección de datos de las encuestas. Se identificaron elementos con influencia significativa no favorable que impactan en el emprendedor de la FCP de la BUAP, lo anterior como consecuencia natural del diseño de la currícula de la Facultad y los retos actuales que desafían la formación del emprendedor.

Por todo lo anterior, encontrar estas áreas de oportunidad permiten hacer los cambios necesarios a las materias económico administrativas con el objetivo de fortalecer el contexto que deben conocer los estudiantes acerca del ecosistema emprendedor y así mejorar sensiblemente su perfil emprendedor.

\subsection{La propuesta}

Derivado de las anteriores respuestas y el planteamiento de los emprendedores en tiempo COVID se puede observar que se debe reforzar la currícula en materia del ecosistema emprendedor para que los estudiantes puedan discernir el contexto mundial, porque más de la mitad requiere enfocar mejor el entorno emprendedor.

Es un hecho que esta propuesta conlleva a una segunda fase de investigación para los ajustes correspondientes a la currícula de la Facultad de Contaduría Pública de la Benemérita Universidad Autónoma de Puebla

Por lo tanto, la propuesta está estructurada en 4 ejes, tal como se visualiza en la figura 13:

Figura 13. Propuesta para fortalecer la percepción del impacto de las materias económico-administrativas en el emprendimiento, caso FCP BUAP.

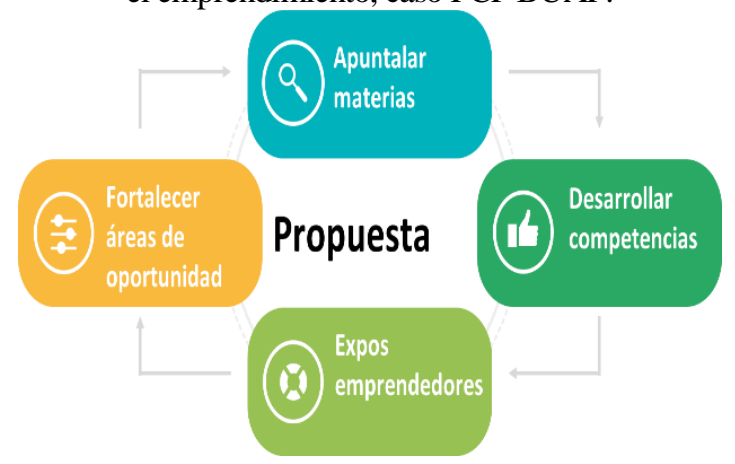

Fuente. Elaboración propia

Apuntalar materias. Las materias que se enfocan a apuntalar el espíritu emprendedor en la Facultad de Contaduría Pública son Administración, Finanzas, Economía de la empresa, Diseño y emprendimiento de negocios y Start Ups, mismas que en sus programas de asignatura contemplan el termino de emprendedor, características, plan de negocios, economía de la empresa, proceso administrativo, así como el desarrollo de Start Ups. 
Desarrollo de competencias. Las competencias del emprendedor combinan la creatividad, el sentido de la iniciativa, la resolución de problemas, la capacidad de reunir recursos y el conocimiento financiero y tecnológico. Estas competencias permiten a los emprendedores y empleados emprendedores provocar y adaptarse al cambio. Dado que es un imperante de la consolidación del conocimiento el tener desarrolladas competencias tales como dominio de las TICs, trabajo en equipo, liderazgo, comunicación asertiva, adaptabilidad al cambio, hacen frente a una mejor inserción laboral en el mundo del emprendimiento dotando no sólo de las mejores herramientas sino de los atributos que le permitan desarrollar acción efectiva en su quehacer.

Fortalecer áreas de oportunidad. Robustecer el contexto que deben conocer los estudiantes acerca del ecosistema emprendedor, esto es, dotar de todas las herramientas que facilitan el surgimiento de empresas y proyectos empresariales comprendiendo las nuevas variables a considerar tales como él E-Commerce, la sostenibilidad ambiental, los retos de género y demográficos que generen un bienestar social y donde la innovación, la creatividad y el emprendimiento resultan fundamentales.

Expos emprendedores. En el 2006 se ofertó la materia de emprendedores que era de carácter optativo y en 2009 se implementó junto con el nuevo plan de estudios una materia denominado Innovación y talento emprendedor en la que se resaltó la importancia de la detección de talento y el generar un producto, proceso o servicio innovador mismo que al final la materia debía presentarse en el evento denominado expo emprendedores, evento en el cual los estudiantes mostraban a toda la población estudiantil su proyecto de emprendimiento con innovación, la expo emprendedores funcionaba como un concurso en el que los proyectos eran expuestos y defendidos por sus creadores ante expertos que fungían como jueces. Se privilegiaba a los proyectos con impacto social que cumplieran con triple o cuádruple hélice. Esta estrategia funcionó, sin embargo, no con los resultados esperados porque aun cuando muchos de los proyectos eran buenos, pocos se llevaron al campo de lo tangible, además de que se les hacía la recomendación de que continuarán trabajando con sus proyectos en la incubadora de empresas de la Institución, pocos alumnos eran los que continuaban en esta labor. Lo rescatable de esa experiencia es que los alumnos adquirían práctica en un entorno de aprendizaje más estructurado, es decir, cumplió con algunos de sus cometidos. La Facultad de Contaduría Pública ya ha tenido experiencia de que a través de los expos emprendedores se genera un vínculo entre docentes, estudiantes y emprendedores exitosos, ahora en este trabajo se propone que nuevamente se realice este evento en el que se cuente con la participación de los funcionarios públicos de la secretaría de economía, servicio de administración tributaria, y todas las dependencias que apoyen programas de emprendimiento

\section{CONCLUSIONES}

El emprendedor es un líder natural que es resultado de la cultura del esfuerzo hasta convertirse en un gran líder que sus trabajadores y empleados lo consideran un ejemplo a seguir. El emprendedor exitoso en cuanto empieza a crecer inicia con pocos apoyos de teoría económica o administrativa, pero al poco tiempo reconoce la necesidad de aplicar y asesorarse de la teoría económica, contable, administrativa y fiscal.

Por un lado, consideramos que la participación activa de los profesores en el rediseño de planes de estudio permita 
garantizar el desarrollo de las competencias de los emprendedores, lo cual conlleve a tan anhelado éxito, sin morir en el intento. Las instituciones de educación superior pueden mejorar aún más sus procesos educativos y es un compromiso constante de nuestra propia Universidad.

Por otro lado, el desarrollo de una cultura del emprendimiento en la sociedad es una herramienta básica para el desarrollo económico y social que mejora la competitividad de un país. Está ampliamente demostrado que el emprendimiento es uno de los ejes del proceso económico. Los emprendedores, a través de la creación de nuevas actividades, promueven el desarrollo de la economía. De ahí que el Estado debe considerar a los emprendedores como aliados en la creación de empleos, por lo tanto, debe generar una política fiscal que favorezca a los emprendedores que están creando empleos y aportando al progreso económico. 


\section{REFERENCIAS}

Alcaraz, R. (2011). El emprendedor de éxito. McGraw-Hill.

Arancibia, C. A. (2021). El Fomento de la Cultura Emprendedora en el proceso de la Formación Universitaria dentro del Ecosistema Emprendedor. Ciencia Latina Revista Científica Multidisciplinar, 5(2), 1926-1950.

Asociación Nacional de Universidades e Instituciones de Educación Superior. (2016). Plan de Desarrollo Institucional. Visión 2030. México: ANUIES.

Herrera, C. E., \& Montoya, L. A. (2013). El emprendedor: una aproximación a su definición y caracterización. Punto de vista, 4(7).

Kao, J. J. (1989). Entrepreneurship, creativity \& organization. Prentice Hall.

Mababu-Mukiur, R. (2017). La transformación digital y el emprendimiento de los jóvenes en Iberoamérica. Relaciones Laborales y Derecho del Empleo, 5(2), 1-17.

Schollhammer H. (1980) Analysis and Assessment of internal corporate entrepreneurship strategies, Los Angeles, C.A., Graduate School of Management.

Organización Mundial de la Salud (2021). COVID time line. Recuperado de: https://www.who.int/es/news/item/29-06-2020-covidtimeline 